\title{
Fertility-sparing management in cervical cancer: balancing oncologic outcomes with reproductive success
}

\author{
Karla Willows ${ }^{1}$, Genevieve Lennox ${ }^{1}$ and Allan Covens ${ }^{1,2^{*}}$
}

\begin{abstract}
Background: Cervical cancer is the fourth most common cancer among women worldwide, many of who are still within their reproductive lifespan. Advances in screening and treatment have increased the 5-year survival for early stage disease to over $90 \%$ in developed countries. The focus is now shifting to reducing morbidity and improving fertility outcomes for cervical cancer patients. Radical trachelectomy with lymph node assessment became the standard of care for selected women with lesions $<2 \mathrm{~cm}$ who desire fertility preservation. However, several questions still remain regarding the degree of surgical radicality required for tumors $<2 \mathrm{~cm}$, and fertility-sparing options for women with early-stage disesase $\geq 2 \mathrm{~cm}$, and those with more advanced disease. Here, we compile a narrative review of the evidence for oncologic and pregnancy outcomes following radical trachelectomy, nonradical fertility-sparing surgery, and the use of neoadjuvant chemotherapy prior to surgery for larger lesions. We also review the literature for assisted reproductive technologies in women with more advanced disease.
\end{abstract}

Findings: Available literature suggests that the crude recurrence and mortality rates after radical trachelectomy are $<5$ and $<2 \%$, respectively (approx. 11 and $4 \%$ for tumors $\geq 2 \mathrm{~cm}$ ). Among 1238 patients who underwent fertilitysparing surgery for early cervical cancer there were 469 pregnancies with a $67 \%$ live birth rate. Among 134 cases with lesions $\geq 2 \mathrm{~cm}$, there were ten conceptions with a live birth rate of $70 \%$. Outcomes after non-radical surgery (simple trachelectomy or cervical conization) are similar, although only applicable among a highly selected patient population. For patients ineligible for fertility-preserving surgery or who require adjuvant radiation therapy, current options include ovarian transposition and cryopreservation of oocytes or embryos but other techniques are under investigation.

Conclusion: Today, many cervical cancer survivors have successful pregnancies. For those with early-stage disease, minimally invasive and fertility sparing techniques have resulted in improved obstetrical outcomes without compromising oncologic safety. Results from three ongoing trials on non-radical surgery for low-risk tumors $<2 \mathrm{~cm}$ will further inform the need for radical surgery in such patients. For those in whom natural childbearing is unachievable, advances in assisted reproductive technologies provide reproductive options. Despite our advances, the effects of cervical cancer survivorship on quality of life are not fully elucidated.

Keywords: Fertility-sparing, Cervical cancer, Trachelectomy, Non-radical, Neoadjuvant chemotherapy, Assisted reproductive technologies, Quality of life,

\footnotetext{
*Correspondence: al.covens@sunnybrook.ca

${ }^{1}$ Division of Gynecologic Oncology, Department of Obstetrics and

Gynecology, University of Toronto, M700-610 University Avenue, Toronto

M5G 2 M9, ON, Canada

${ }^{2}$ Division of Gynecologic Oncology, T2051 Odette Cancer Centre, University

of Toronto, 2075 Bayview Avenue, Toronto M4N 3 M5, ON, Canada
} 


\section{Background}

Cervical cancer is the fourth most common cancer in women worldwide, with over half a million new cases diagnosed annually [1]. It affects women at a significantly younger age than most other malignancies. According to the Surveillance, Epidemiology, and End Results (SEER) database, between 2008 and 2012, 39 \% of new cases diagnosed in the US were in women under the age of 45 [2]. Over the past several decades, most developed countries have seen a significant reduction in overall mortality with 5 -year survival rates for localized disease surpassing $90 \%$ [2]. Combined with a trend towards delayed childbearing, this has resulted in a cohort of cervical cancer survivors who are still well within their reproductive lifespan.

Loss of fertility, regardless of cause, is a source of significant psychological distress among women [3]. Several studies suggest that cervical cancer survivors have significantly more reproductive concerns, compared to age-matched controls, including grief about inability to bear children, and an inability to talk openly about fertility [3, 4]. In 2006 the American Society of Clinical Oncology highlighted the importance of addressing future fertility and potential fertility preservation options with patients prior to cancer therapy [5].

Radical trachelectomy (RT) was first described by Eugen Aburel for the treatment of early-stage cervical cancers in the 1950s [6]. This technique was all but forgotten until the 1990s when it was revitalized by Dargent et al. in 1994 [7], to preserve fertility in selected cases through a vaginal approach (VRT). This ushered in a new era of fertility-sparing options for women with early-stage cervical cancer. Over two decades of accumulated data show that for women with small volume disease, this procedure has acceptable surgical morbidity and oncological outcomes [8]. As a result, radical trachelectomy, with pelvic lymph node assessment, became the standard of care for selected women early-stage with disease $<2 \mathrm{~cm}$ who desire to maintain their fertility [9].

However, several questions still remain about the degree of radicality required, as well as the optimal management of lesions greater than $2 \mathrm{~cm}$. There continues to be a push towards less invasive procedures to reduce peri-operative morbidity, and reduce preterm delivery and perinatal morbidity without compromising oncologic safety. The purpose of this review is to examine the current state of fertility sparing management of cervical cancer, including management of $\geq 2 \mathrm{~cm}$ early stage disease and novel technologies in assisted reproduction for women with locally advanced disease.

\section{Main text}

\section{Methods}

We searched Ovid EMBASE (from 1974 to 2016 week 13) and Ovid MEDLINE in process \& other non-indexed citations (from inception to March 2016) for relevant citations. We performed key-word searches combining various disease-specific terms (e.g. cervical cancer, uterine cervix carcinoma) with treatment specific terms (e.g. trachelectomy, conization, neoadjuvant chemotherapy). We limited our search to English language studies. To identify ongoing planned or unpublished trials, we searched the US National Institute of Health's clinical trial registry at ClinicalTrials.gov. All searches were supplemented by hand searching the reference lists of key papers for relevant citations. Articles were organized based on topics deemed to be relevant by the authors. Crude recurrence, mortality, and birth rates were calculated from large reports of radical trachelectomy overall, radical trachelectomy for lesions $\geq 2 \mathrm{~cm}$, non-radical fertility sparing procedures, and fertility-sparing procedures after neoadjuvant chemotherapy. Where follow-up studies were available, we included only the most recent and complete series to avoid double counting patients. A narrative review of these topics is presented here.

\section{Early-stage disease}

Early-stage cervical cancer includes disease that is confined to the cervix, measuring $\leq 4 \mathrm{~cm}$, with no apparent spread to adjacent structures or distant organs (International Federation of Gynecology and ObstetricsFIGO- stages IA1-IB1) [10]. Given the low risk ( $\leq 1 \%)$ of either pelvic lymph node or parametrial involvement in stage 1A1 squamous cell carcinoma of the cervix, standard treatment usually consists of cone biopsy or extrafascial hysterectomy, depending on the patient's desire for fertility preservation [10, 11]. Traditional thinking has dictated that beyond stage IA1 (or in the presence of other high risk features) the increased risk of local spread to the parametria and upper vagina necessitates a more radical surgical approach including lymph node assessment.

Excellent oncological outcomes have been obtained with radical hysterectomy accompanied by bilateral pelvic lymph node dissection for early-stage disease. Five-year overall survival rates range from 73 to $98 \%$ [12-14]. However, this procedure carries a significant risk of surgical morbidity, including increased blood loss, transfusion, and injuries to the bladder, bowel, ureters, and obturator nerve [15-17]. Long-term bladder, anorectal, and sexual dysfunction have been described [18-20]. Over time, minimally invasive and nerve sparing approaches have been developed to reduce morbidity [10], and the degree of surgical radicality required has been challenged with favourable survival and recurrence rates [21, 22].

\section{Eligibility for fertility sparing management}

To be eligible for fertility sparing management of cervical cancer, two main criteria must be met; 1) the 
patient's desire for, and likelihood of, fertility must be sufficiently high, and 2) oncologic safety must be acceptable. A pre-operative fertility workup may be considered, particularly in those with a history of infertility [23]. The patient's age should be considered with respect to ovarian reserve and the increased risk of pregnancy complications with advanced maternal age [23, 24]. Eligibility for fertility-sparing surgery should reflect local jurisdicational age-related eligibility policies for assisted reproductive technologies.

Oncologic safety can be assessed in terms of clinicopathologic risk factors for recurrence. These include tumour size, depth of stromal invasion, presence of lymphovascular space invasion (LVSI), lymph node and parametrial involvement and the feasibility of achieving tumour-free margins [25-27].

Clinical stage, including vaginal and parametrial spread, should be assessed by an expert (e.g. Gynecologic Oncologist) [23]. Although tumours $>2 \mathrm{~cm}$ are at greater risk of lymph node metastasis and recurrence $[28,29]$, there is a general consensus that larger, expohytic tumours with minimal stromal invasion may be considered for fertility-sparing procedures [30, 31]. In a small prospective trial of 30 patients desiring fertilitypreservation, magnetic resonance imaging was shown to have $100 \%$ positive and negative predictive value in assessing suitability for radical trachelectomy [32].

For early stage disease, the difference in recurrence and mortality between squamous carcinomas and adenocarcinomas seems negligible, and both may be considered as candidates for fertility-preservation [33, 34]. A review at our centre found no significant difference in recurrence free survival between 74 patients with adenocarcinoma and 66 patients with squamous cell carcinoma treated with radical vaginal trachelectomy [35]. Although there have been reports of higher incidence of ovarian involvement in adenocarcinoma of the cervix, compared to squamous carcinomas, the overall risk remains low. A review by Touhami and Plante reported a $2 \%$ incidence of ovarian metastasis among those with stage $1 \mathrm{~B}$ adenocarcinomas of the cervix, among which $96.7 \%$ had other clinical and pathologic features that would preclude fertility-sparing [36]. The authors argue that the risk of surgical menopause in the premenopausal population eligible for fertility-sparing outweighs the risk of ovarian involvement, and therefore advocate that ovarian preservation remains an option in the case of early adenocarcinomas of the cervix.

However, non-squamous, non-adenocarcinomas have significantly worse prognosis [37]. In a few early series that included neuroendocrine carcinomas for fertilitysparing, rapid recurrence was observed [28-30]. Many have thus questioned the inclusion of these aggressive histologies in fertility sparing procedures. LVSI alone should not preclude fertility-sparing management. A review by Beiner et al. found that among patients undergoing radical vaginal trachelectomy, $28 \%$ had LVSI, and only $5 \%$ had nodal metastases [38]. While exclusion on this basis is unmerited, extensive LVSI does put these patients at increased risk of nodal involvement [39].

Little prospective data exists as to the optimal surgical margin for trachelectomy specimen. A retrospective review by McCann et al. found that for patients with stage 1A2-2A cervical cancer undergoing radical hysterectomy, close surgical margins (defined as margins $\leq 5 \mathrm{~mm}$ ), while not an independent risk factor for recurrence, were associated with other intermediate and high risk features, including lymph node positivity, parametrial involvement, increased size of primary lesion, increased depth of stromal invasion and LVSI [40]. Coincidently, most experts in the area had previously empirically adopted $5 \mathrm{~mm}$ as the minimum margin $[41,42]$. Therefore, based on extrapolation of data on recurrence following radical hysterectomy and the above empiricism, we believe that optimal surgical margins after fertility-sparing management are at least $5 \mathrm{~mm}$.

\section{Lymph node assessment}

Assessment for lymph node metastases is critical for any patient with greater than $3 \mathrm{~mm}$ depth of invasion (i.e. > stage IA1) or other high risk features (e.g. LVSI, highrisk histologies) on a biopsy specimen. Suspicious nodes should be sought on pre-operative CT or MRI but the sensitivity and specificity of these modalities in early cervical cancer is modest, given the low prevalence of enlarged nodes in this population [11]. Combined PET-CT may identify small metastases, however the utility and significance of these remains controversial, particularly in those receiving neoadjuvant chemotherapy prior to surgical management [43].

In the absence of grossly positive nodes on imaging, definitive nodal assessment must be made operatively. Frozen section may be utilized to assess for nodal metastases and positive surgical margins, in which case fertility-sparing surgery may be aborted, or the surgical procedure altered (eg. complete ipsilateral pelvic and para-aortic lymphadenectomy). However, frozen section is not universally practiced due to the concerns regarding false negatives, and loss of tissue for permanent pathological processing. A range of false negative rates for intra-operative frozen section in early cervical cancer has been reported. For stage 1A2-1B1, Panici et al. report a false negative rate of $4.2 \%$ [44]; for stage 1B1-2B, Scholz et al. report a false negative rate of $19 \%$ [45]. Since 2015, the National Comprehensive Cancer Network (NCCN) recommends the consideration of sentinel lymph node procedure (SLNP) for early-stage cervical cancer measuring less than $2 \mathrm{~cm}$ [9]. Gortzak 
examined the use of sentinel lymph node procedure among 81 women undergoing successful sentinel lymph node procedure for early cervical cancer (stage 1A-1B1). They reported a false negative rate of $21.4 \%(3 / 14$ negative sentinel nodes). Two of the three cases involved micrometastases $<2 \mathrm{~mm}$ found only after ultrastaging, highlighting the importance of this element of the sentinel lymph node procedure [46]. Despite a high false negative rate, intraoperative frozen section remains useful in this setting, due to the low prevalence of nodal involvement in early cervical cancer. In this case, the finding of a negative node on frozen section has a negative predictive value of over $97 \%[47,48]$.

It is recommended that both intraoperative, and final pathology be reviewed by a pathologist specializing in gynecology and that ultrastaging be performed for sentinel lymph nodes. Ultimately, the clinician needs to evaluate all available information, and come to a decision regarding further surgery to define the extent of disease (staging), versus adjuvant therapy be it chemo, radiation, or both- the former not necessarily precluding fertility sparing.

\section{Radical fertility-sparing surgical management}

Radical vaginal trachelectomy (VRT) accompanied by laparoscopic pelvic lymph node dissection has become an accepted treatment modality for fertility preservation in early cervical cancer measuring $<2 \mathrm{~cm}$. In 2007, a review of 520 cases found a recurrence and mortality rate of 4.2 and $2.8 \%$, respectively [49].

Radical trachelectomy can also be performed abdominally (ART), laparoscopically (LRT), and robotically (RRT). An advantage to these alternative approaches is that they more closely resemble the radical hysterectomy familiar to gynecologic oncologists, and do not require special skills in vaginal surgery [34]. Additionally, an abdominal approach allows for potentially greater parametrial resection compared to the vaginal approach [50]. In 2013, Cao et al. performed a matched case-control study comparing surgical approaches in 126 patients undergoing radical trachelectomy [51]. They found no significant differences between VRT and ART for mean operating time, perioperative complications or postoperative complications. Although VRT resulted in higher pregnancy rates ( 35.5 v. $8.8 \%)$ and live birth rates $(23.3$ v. $8.8 \%$ ), it also resulted in higher rates of recurrence (9.8 v. $0 \%$ ) and death from disease (2.8 v. $0 \%$ ) [51]. Our review of large case series' of radical trachelectomy identified oncologic outcomes in 1312 patients eligible for fertility sparing management of early cervical cancer (Table 1). After accounting for adjuvant treatments, $91 \%$ successfully preserved their fertility. The crude recurrence and mortality rates in this group were 4.5 and $1.7 \%$, respectively. We identified 13 studies that reported individual-level data on recurrences after radical trachelectomy. Fifty-six patients recurred at a median of 18 months after surgery (range 3-108 months). The majority $(66 \%)$ of recurrent cases had evidence of intermediate or high-risk features on surgical pathology, or a non-squamous, non-adenocarcinoma histology (Table 2).

Once tumour-free margins $(>5 \mathrm{~mm})$ have been achieved, many surgeons insert a cerclage suture around the lower uterine segment, in anticipation of future pregnancy [34]. In an attempt to prevent isthmic stenosis (which occurs in approximately $15 \%$ of cases [52]) we suture a rubber catheter into the os of the lower uterine segment. In our center, this is removed 3 weeks post-operatively [34]. Alternatively, some advocate for the routine use of a temporary intrauterine device for this purpose [53]. If stenosis is suspected, cervical dilatation can be performed [52]. Our review of obstetrical outcomes among 1238 patients who had undergone successful fertility-sparing management for early cervical cancer identified 469 pregnancies, resulting in a $67 \%$ crude live birth rate (Table 3).

Regardless of the approach, higher recurrence rates have been found in patients with larger tumours [51]. Our review of the literature identified 189 cases (for which individual-level data was extractable) of lesions $>2 \mathrm{~cm}$ eligible for radical trachelectomy (Table 4). Among those who successfully underwent fertility sparing surgery, we identified an overall crude recurrence rate of $11 \%$ and a crude disease-related mortality rate of $4 \%$. Many feel that lesions $\geq 2 \mathrm{~cm}$ should be triaged to the abdominal approach, where a wider parametrial resection is more attainable $[50,54]$. The use of neoadjuvant chemotherapy in this population is discussed below. Furthermore, we identified 134 cases of lesions $\geq 2 \mathrm{~cm}$ where fertility sparing management was successful, resulting in ten conceptions, with a live birth rate of $70 \%$ (Table 5). Ultimately, approximately $25-30 \%$ of women who try to conceive post radical trachelectomy will be infertile [52]. Although three quarters of cases can be attributed to cervical factor, the remaining cases are due to other causes, highlighting the importance of a pre-operative fertility workup in some cases [52].

\section{Non-radical surgical management}

Parametrectomy is responsible for the majority of complications related to radical surgery [55]. Among a subgroup of patients with low-risk pathologic features (lesion $<2 \mathrm{~cm}$, depth of invasion $<10 \mathrm{~mm}$, and negative pelvic nodes), the risk of paramentrial involvement is estimated to be as low as $0.6 \%$ (90\% CI 0-1.1\%) [56]. Furthermore, after diagnostic LEEP/conization procedures, approximately $65 \%$ of radical trachelectomy specimens have no residual disease [57-59]. Therefore, many patients with early cancers are over-treated at the 
Table 1 Oncologic outcomes after radical trachelectomy (where $N>100$ reported)

\begin{tabular}{|c|c|c|c|c|c|c|c|c|c|c|c|}
\hline Study & $\begin{array}{l}\text { Eligible for } \\
\text { fertility sparing (N) }\end{array}$ & Stage $(\mathrm{N})$ & Histology (N) & LVSI+ (N) & Approach & $\mathrm{LN}+(\mathrm{N})$ & $\begin{array}{l}\text { Required adjuvant } \\
\text { therapy }(\mathrm{N})\end{array}$ & $\begin{array}{l}\text { Successful fertility } \\
\text { sparing }(N)\end{array}$ & $\begin{array}{l}\text { Primary recurrences } \\
\text { (N) (mos) }\end{array}$ & $\begin{array}{l}\text { Dead of disease } \\
\text { (N) (mos) }\end{array}$ & $\begin{array}{l}\text { Median follow } \\
\text { up months } \\
\text { (range) }\end{array}$ \\
\hline $\begin{array}{l}\text { Shepherd } 2006 \\
\text { [108] }\end{array}$ & 123 & $\begin{array}{l}\mid \mathrm{A} 2=2 \\
\mid \mathrm{B} 1=121\end{array}$ & $\begin{array}{l}83 \text { SCC } \\
33 \text { AC } \\
3 \text { AS } \\
4 \text { other }\end{array}$ & 39 & VRT & 7 & 11 & 112 & $5(15,19,21,31,84)$ & $4(26,26,32,32)$ & $45^{a}(1-120)$ \\
\hline $\begin{array}{l}\text { Marchiole } 2007 \\
\text { [109] }\end{array}$ & 118 & $\begin{array}{l}\mid A 1=10 \\
\mid A 2=19 \\
\mid B 1=83 \\
\| A=6\end{array}$ & $\begin{array}{l}90 \mathrm{SCC} \\
25 \mathrm{AC} / \mathrm{AS} \\
3 \text { rare }\end{array}$ & 43 & LAVRT & 5 & 8 & 97 & $\begin{array}{l}7(7,11,18,19,20,21, \\
93)\end{array}$ & $\begin{array}{l}5(21,24,26,27 . \\
41)\end{array}$ & $95(31-234)$ \\
\hline Plante 2011 [57] & 140 & $\begin{array}{l}\mid A 1=7 \\
\mid A 2=30 \\
\mid B 1=97 \\
\mid B 2=3 \\
\| A=3\end{array}$ & $\begin{array}{l}78 \mathrm{SCC} \\
52 \mathrm{AC} \\
10 \mathrm{AS}\end{array}$ & 40 & VRT & 5 & 15 & 110 & $6(-)$ & $2(-)$ & $95(4-225)$ \\
\hline Helpman 2011 [35] & 140 & All IA-IB & 74 AC 66 SCC & 55 & VRT & 8 & 9 & 140 & $8(-)$ & 0 & $60(-)$ \\
\hline $\begin{array}{l}\text { Wethington } 2012 \\
\text { [110] }\end{array}$ & 101 & $\begin{array}{l}\mid \mathrm{A} 1=3 \\
\mid \mathrm{A} 2=8 \\
\mathrm{IB} 1=88 \\
\mathrm{IB} 2=1 \\
\| \mathrm{A}=1\end{array}$ & $\begin{array}{l}40 \text { SCC } \\
6 \text { AS } \\
54 \text { AC } \\
1 \text { clear cell }\end{array}$ & 47 & ART & 19 & 20 & 70 & $4(-)$ & 0 & $32(1-124)$ \\
\hline Cao 2013 [51] & 150 & $\begin{array}{l}18 \mid \mathrm{A} 1 \\
19 \mid \mathrm{A} 2 \\
113 \mathrm{IB} 1\end{array}$ & $\begin{array}{l}135 \mathrm{SCC} \\
15 \mathrm{AC}\end{array}$ & 8 & $\begin{array}{l}\text { VRT } \\
\text { ART }\end{array}$ & 0 & 0 & 150 & $7(-)$ & $2(-)$ & $25(6-91)$ \\
\hline Mangler 2014 [111] & $320^{*}$ & $\begin{array}{l}\mid A 1=46 \\
\mid A 2=68 \\
\mid B 2=207\end{array}$ & $\begin{array}{l}220 \mathrm{SCC} \\
97 \mathrm{AC} \\
5 \mathrm{AS}\end{array}$ & 94 & VRT & - & - & 320 & $\begin{array}{l}10 \text { (mean } 26.1 \\
\text { month, range 3-108) }\end{array}$ & $\begin{array}{l}5(16,19,22,29, \\
30)\end{array}$ & $48(0-216)$ \\
\hline $\begin{array}{l}\text { Hauerberg } 2015 \\
\text { [112] }\end{array}$ & 120 & $\begin{array}{l}\mathrm{CIS}=2 \\
\mid \mathrm{A} 1=7 \\
\mid \mathrm{A} 2=8 \\
\mid \mathrm{B} 1=103\end{array}$ & $\begin{array}{l}82 \mathrm{SCC} \\
36 \mathrm{AC} \\
2 \mathrm{AS}\end{array}$ & 30 & VRT & 4 & 12 & 108 & $6(-)$ & $2(-)$ & $55.7(5.5-147)$ \\
\hline Vieira 2015 [113] & 100 & $\begin{array}{l}\mid A 1=6 \\
\mid A 2=25 \\
\mid B 1=69\end{array}$ & $\begin{array}{l}49 \mathrm{SCC} \\
42 \mathrm{AC} \\
7 \mathrm{AS} \\
2 \text { mixed }\end{array}$ & 25 & $\begin{array}{l}\text { ART } \\
\text { RRT } \\
\text { LRT }\end{array}$ & 2 & 9 & 83 & 0 & 0 & $51(10-147)$ \\
\hline Total & $N=1312$ & & & & & & & $N=1190$ & $N=53$ & $N=20$ & \\
\hline Crude rates (\%) & & & & & & & & & $\begin{array}{l}\text { Recurrence rate }= \\
4.5 \%{ }^{\#}\end{array}$ & $\begin{array}{l}\text { Mortality rate }= \\
1.7 \%^{\#}\end{array}$ & \\
\hline
\end{tabular}

Abbreviations: LVSI+ presence of lymphovascular space invasion, LN+ lymph node metastasis, SCC squamous cell carcinoma, AC adenocarcinoma, AS adenosquamous carcinoma, LAVRT laparoscopic-assisted vaginal radical trachelectomy, ART abdominal radical trachelectomy, VRT vaginal radical trachelectomy, RRT robotic radical trachelectomy

anly mean follow up is reported

"Crude recurrence and mortality rates among those who successfully had fertility preservation

*In the original study, the sum of the stages and histologies are 321 and 322, respectively, but the reported $N$ is 320 
Table 2 Histopathologic features among recurrences after radical trachelectomy $(N=56)$

\begin{tabular}{lc}
\hline Intermediate/high risk features & $N$ \\
\hline Histology & \\
Squamous cell carcinoma & 26 \\
Adenocarcinoma & 18 \\
Adenosquamous & 4 \\
Clear cell & 1 \\
Neuroendocrine & 2 \\
Glassy cell & 1 \\
Not reported & 4 \\
Size & \\
Z2 cm & 20 \\
Lymphovascular space invasion & \\
positive & 22 \\
Lymph nodes & \\
positive & 8 \\
Margins & \\
$\quad$ positive & \\
No intermediate/high risk features & 19 \\
\hline
\end{tabular}

risk of increased surgical morbidity without the benefit of improved oncologic outcomes. A review by Reade et al. identified 341 patients who had undergone simple hysterectomy or simple trachelectomy for the treatment of stage $\geq$ IA 2 cervical cancer. They found a crude recurrence rate of $6.3 \%$, and a crude disease-related mortality rate of $1.5 \%$ [11], which are comparable to those achieved by radical trachelectomy $[49,51]$. Given these findings, non-radical surgery (simple trachelectomy or conization) could be considered for fertility-sparing in the management of small lesions with favourable prognostic features $[55,56,60]$.

Our review of the literature identified 203 cases of early-stage cervical cancer eligible for non-radical, fertility-sparing surgery (Table 6). All patients had lesions $<2 \mathrm{~cm}$. Sixty patients underwent simple trachelectomy, and 138 underwent conization. Among 185 cases where fertility-sparing was successful, the crude recurrence rate was $2.7 \%$ and the crude mortality rate was $0.5 \%$. Among 124 women where fertility preservation was successful, we identified 71 pregnancies with a live birth rate of $68 \%$ (Table 7). Both oncologic outcomes and pregnancy rates compare favourably to literature reports of those undergoing radical trachelectomy. However, it should be noted that the available data is from non-randomized studies of highly selected patient populations with more favourable prognostic factors compared to those undergoing radical surgery.

We identified three ongoing prospective trials designed to assess the efficacy of non-radical surgery in the treatment of low-risk early-stage cervical cancer. The SHAPE trial (NCT01658930) is a randomized trial comparing simple hysterectomy to radical hysterectomy (or cone biopsy to radical trachelectomy) in addition to pelvic lymph node assessment for cases of early-stage

Table 3 Obstetrical outcomes after radical trachelectomy (where $N>50$ reported)

\begin{tabular}{|c|c|c|c|c|c|c|}
\hline Study & $\begin{array}{l}\text { Successful fertility } \\
\text { sparing management }\end{array}$ & $\begin{array}{l}\text { Attempted } \\
\text { to conceive }\end{array}$ & Conceptions & T1/T2 losses & $\begin{array}{l}\text { Live births } \\
\text { (ongoing pregnancy) }\end{array}$ & $\begin{array}{l}\text { Median follow up } \\
\text { months for entire } \\
\text { series (range) }\end{array}$ \\
\hline Bernardini 2003 [114] & 80 & 39 & 22 & 4 & 18 & - \\
\hline Hertel 2006 [29] & 106 & - & 18 & 3 & $12(3)$ & $29(1-128)$ \\
\hline Shepherd 2006 [108] & 112 & 63 & 55 & - & $28(3)$ & $45^{\mathrm{a}}(1-120)$ \\
\hline Li 2011 [53] & 56 & 10 & 2 & 0 & $1(1)$ & $23(1-78)$ \\
\hline Plante 2011 [57] & 110 & - & 106 & 29 & 77 & $95(4-225)$ \\
\hline Kim 2012 [115] & 77 & 35 & 27 & 7 & 20 & - \\
\hline Wethington 2012 [110] & 70 & 38 & 31 & 9 & $16(6)$ & $32(1-124)$ \\
\hline Cao 2013 [51] & 150 & 77 & 20 & 9 & 14 & $25(6-91)$ \\
\hline Nishio 2013 [116] & 114 & 69 & 31 & 5 & $21(5)$ & 33 \\
\hline Vieira 2015 [113] & 83 & 34 & 19 & 5 & $10(4)$ & $51(10-147)$ \\
\hline Hauerberg 2015 [112] & 108 & 72 & 77 & 21 & $53(3)$ & $55.7(5.5-147)$ \\
\hline Kasuga 2016 [117] & 172 & 109 & 61 & 13 & $43(5)$ & - \\
\hline Total & 1238 & 546 & 469 & 105 & $313(30)$ & \\
\hline $\begin{array}{l}\text { Crude rates } \\
(\%)\end{array}$ & & & & $\mathrm{TI} / \mathrm{T} 2$ loss rate $=22.4 \%$ & Live birth rate $=66.7 \%$ \& & \\
\hline
\end{tabular}

Abbreviations: $T 1$ first trimester, $T 2$ second trimester

${ }^{a}$ Only mean follow up is reported

"Excludes those who had completion hysterectomy, or received fertility-compromising adjuvant treatment

\& Does not include ongoing pregnancies 
Table 4 Oncologic outcomes of radical trachelectomy for lesions $\geq 2 \mathrm{~cm}$ (where $N>10$ reported)

\begin{tabular}{|c|c|c|c|c|c|}
\hline Study & $\begin{array}{l}\mathrm{N} \text { with lesions } \geq 2 \mathrm{~cm} \\
\text { who underwent fertility } \\
\text { sparing surgery }\end{array}$ & Approach & $\mathrm{N}$ recurrences (mos) & $\begin{array}{l}\mathrm{N} \text { dead of } \\
\text { disease (mos) }\end{array}$ & $\begin{array}{l}\text { Median follow up } \\
\text { months for entire } \\
\text { series (range) }\end{array}$ \\
\hline Marchiole 2007 [109] & 21 & LAVRT & $6(7,11,18,20,21,93)$ & $4(21,24,27,41)$ & $95(31-234)$ \\
\hline Nishio 2009 [118] & 13 & ART & $5(4,8,14,18,23)$ & 0 & $27(1-67)$ \\
\hline Cao 2013 [51] & 48 & $\begin{array}{l}\text { VRT } \\
\text { ART }\end{array}$ & $5(-)$ & $2(-)$ & $34.3^{\mathrm{a}}$ \\
\hline Li 2013 [119] & 61 & ART & 0 & 0 & $30(2-108)$ \\
\hline Lintner 2013 [120] & 31 & ART & $4(5,6,10,14)$ & $2(16,22)$ & $90(60-148)$ \\
\hline Wethington 2013 [121] & 15 & $\begin{array}{l}\text { ART } \\
\text { LAVRT } \\
\text { VRT }\end{array}$ & $1(9)$ & 0 & $44(1-90)$ \\
\hline Total & $N=189$ & & $N=21$ & $N=8$ & \\
\hline Crude rates (\%) & & & Recurrence rate $=11.1 \%{ }^{\#}$ & Mortality rate $=4.2 \%{ }^{\#}$ & \\
\hline
\end{tabular}

Abbreviations: LAVRT laparoscopic-assisted vaginal radical trachelectomy, ART abdominal radical trachelectomy, VRT vaginal radical trachelectomy

${ }^{a}$ Only mean follow up is reported

${ }^{\#}$ Crude recurrence and mortality rates among those who successfully underwent fertility sparing surgery, notwithstanding adjuvant treatment received

(IA2-IB1 $<2 \mathrm{~cm}$ ), low-risk (stromal invasion $<10 \mathrm{~mm}$ on LEEP/cone, or $<50 \%$ on MRI) cervical cancer [61]. The goal is to demonstrate that in selected cases, non-radical surgery (simple hysterectomy or cone biopsy) is non-inferior to the gold standard radical surgery (radical hysterectomy or radical trachelectomy) with respect to oncologic safety. Treatment-related morbidity, quality of life, and cost-effectiveness are also being evaluated. ConCerv (NCT01048853) is a prospective, international, multicenter cohort study. The goal is to assess the oncologic safety and feasibility of simple hysterectomy or cone biopsy for early-stage (IA2-IB1 $<2 \mathrm{~cm}$ ) low-risk (negative LVSI, negative margins on cone specimen) cervical cancer [62]. GOG 278 (NCT01649089) is a large prospective cohort study [63]. This study's primary objectives are to examine the changes before and after non-radical surgical treatment (simple hysterectomy or cone biopsy for fertility preservation plus pelvic lymphadenectomy) on functional outcomes of bladder, bowel and sexual function for early stage cervical cancer. Women with stage IA1 (LVSI+) and IB1 $(<2 \mathrm{~cm})$ carcinoma of the cervix with $\leq 10 \mathrm{~mm}$ of invasion on diagnostic pathology are eligible for entry. After a preoperative survey on quality of life, women are stratified based on desire to preserve fertility. Those desiring fertility preservation undergo conization, whereas those not desiring future fertility undergo extrafascial hysterectomy. All patients undergo pelvic lymphadenectomy. Patients with high-risk features on final pathology are offered appropriate adjuvant treatment, and are followed for survival only. Otherwise, patients undergoing non-radical (simple hysterectomy) and fertilitysparing (conization) surgery are assessed at routine post-operative visit and every 6 months there-after for validated quality of life measures, including surgical morbidity, sexual function, fertility intentions, reproductive concerns and impact of therapeutic choice overall. Efficacy (recurrence) is an important secondary objective.

It is hoped that these trials will help to define a select group of patients for whom non-radical surgical management is oncologically safe.

Table 5 Obstetrical outcomes of radical trachelectomy for lesions $\geq 2 \mathrm{~cm}$ (where $N>10$ reported)

\begin{tabular}{lllllll}
\hline Study & $\begin{array}{l}\text { Successful fertility } \\
\text { sparing management }{ }^{\#}\end{array}$ & $\begin{array}{l}\text { Attempted } \\
\text { to conceive }\end{array}$ & Conceptions & T1/T2 loss & $\begin{array}{l}\text { Live births } \\
\text { (ongoing) }\end{array}$ & $\begin{array}{l}\text { Median follow up } \\
\text { months for entire } \\
\text { series (range) }\end{array}$ \\
\hline Cao 2013 [51] & 48 & 24 & 3 & 0 & 3 & $34.3^{\text {a }}$ \\
Li 2013 [119] & 55 & 9 & 3 & 2 & 1 & $30.2(2-108)$ \\
Lintner 2013 [120] & 31 & 8 & 4 & 1 & 3 & $90(60-148)$ \\
Total & 134 & 41 & 10 & 3 & T1/T2 loss rate $=30 \%$ & Live birth rate $=70 \%$ \\
$\begin{array}{llll}\text { Crude rates } \\
\text { (\%) }\end{array}$ & & & & &
\end{tabular}

Abbreviations: $T 1$ first trimester, $T 2$ second trimester

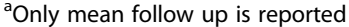

\#Excludes those who had completion hysterectomy, or received fertility-compromising adjuvant treatment 
Table 6 Oncologic outcomes of non-radical fertility sparing procedures (where $N>10$ reported)

\begin{tabular}{|c|c|c|c|c|c|c|}
\hline Study & N eligible & $\begin{array}{l}\text { Surgical procedure } \\
\text { (includes pelvic } \\
\text { LN assessment) }\end{array}$ & $\begin{array}{l}\text { Successful fertility } \\
\text { sparing surgery }\end{array}$ & $\begin{array}{l}N \text { recurrences } \\
\text { (mos) }\end{array}$ & $\begin{array}{l}\mathrm{N} \text { dead of } \\
\text { disease (mos) }\end{array}$ & $\begin{array}{l}\text { Median follow up } \\
\text { months for entire } \\
\text { series (range) }\end{array}$ \\
\hline Bisseling 2007 [122] & 18 & 18 cone & 18 & 0 & 0 & $72^{a}$ \\
\hline Rob 2007 [123] & 26 & $\begin{array}{l}15 \mathrm{ST} \\
7 \text { cone }\end{array}$ & 20 & $1(14)$ & 0 & $49(18-84)$ \\
\hline Landoni 2007 [124] & 11 & 11 cone & 11 & 0 & 0 & $20(7-29)$ \\
\hline Fagotti 2011 [125] & 17 & 17 cone & 13 & 0 & 0 & $16(8-101)$ \\
\hline Maneo 2011 [126] & 36 & 36 cone & 31 & $3(20,34,36)$ & $1(72)$ & $66(18-168)$ \\
\hline Raju 2012 [127] & 15 & $15 \mathrm{ST}$ & 15 & 0 & 0 & $96(12-120)$ \\
\hline Palaia 2012 [128] & 14 & $14 \mathrm{ST}$ & 14 & 0 & 0 & $38(18-96)$ \\
\hline Plante 2013 [129] & 16 & $16 \mathrm{ST}$ & 16 & 0 & 0 & $27(1-65)$ \\
\hline Andikyan 2014 [130] & 10 & $\begin{array}{l}9 \text { cone } \\
1 \mathrm{cx} \mathrm{bx}\end{array}$ & 9 & 0 & 0 & $17(1-83)$ \\
\hline Bouchard-Fortier 2014 [55] & 29 & 29 cone & 29 & 0 & 0 & $21(1-112)$ \\
\hline Salihi 2015 [131] & 11 & 11 cone & 9 & $1(40)$ & 0 & $58(13-122)$ \\
\hline Total & 203 & $\begin{array}{l}138 \text { Cone } \\
60 \text { ST }\end{array}$ & 185 & $N=5$ & $N=1$ & \\
\hline Crude rates (\%) & & & & Recurrence rate $=2.7 \%^{\#}$ & Mortality rate $=0.5 \%{ }^{\#}$ & \\
\hline
\end{tabular}

Abbreviations: ST simple trachelectomy, cx bx cervical biopsy

${ }^{a}$ Only mean follow up is reported

${ }^{\#}$ Crude recurrence and mortality rates among those who successfully underwent fertility sparing surgery, notwithstanding adjuvant treatment received

Bulky $(2-4 \mathrm{~cm})$ early-stage disease and the use of neoadjuvant chemotherapy

For 2-4 cm FIGO stage IB1 and IIA disease, neoadjuvant chemotherapy (NACT) has been shown to reduce nodal metastases, parametrial infiltration, and overall tumour size, theoretically making otherwise unresectable (for fertility preserving purposes) disease amenable to surgical management [64-66]. Although meta-analysis of the available data has yet to show a survival advantage for the use of NACT in early cervical cancer, its use in the context of fertility preservation has been gaining attention [66].

Our literature review identified 80 cases of $\geq 2 \mathrm{~cm}$ stage IB1-IIA disease eligible for NACT prior to fertility-sparing surgery (Table 8 ). The crude recurrence rate is $6.3 \%$ and one patient died from her recurrent disease. The use of NACT has resulted in at least 36 pregnancies with a $72.2 \%$ live birth rate (Table 9).

The timing of nodal assessment with respect to NACT is not standardized. A study by Vercillino et al. in 2012

Table 7 Obstetrical outcomes of non-radical fertility sparing procedures (where $N>10$ reported)

\begin{tabular}{llllll}
\hline Study & $\begin{array}{l}\text { Successful fertility } \\
\text { sparing management }\end{array}$ & Conceptions & T1/T2 losses & $\begin{array}{l}\text { Live births } \\
\text { (ongoing) }\end{array}$ & $\begin{array}{l}\text { Median follow up } \\
\text { months for entire } \\
\text { series (range) }\end{array}$ \\
\hline Bisseling 2007 [122] & 18 & 18 & 5 & 13 & $72^{\text {a }}$ \\
Rob 2007 [123] & 20 & 15 & 6 & $8(1)$ & $49(18-84)$ \\
Landoni 2007 [124] & 11 & 3 & 0 & 3 & $20(7-29)$ \\
Fagotti 2011 [125] & 13 & 2 & 0 & 2 & $16(8-101)$ \\
Maneo 2011 [126] & 31 & 21 & 6 & $14(1)$ & $66(18-168)$ \\
Raju 2012 [127] & 15 & 4 & 0 & 4 & $96(12-120)$ \\
Plante 2013 [129] & 16 & 8 & 0 & $48(4)$ & $27(1-65)$ \\
Total & 124 & 71 & 17 & T1/T2 loss rate $=23.9 \%$ & Live birth rate $=67.6 \% \&$ \\
Crude rates & & & & &
\end{tabular}

Abbreviations: $T 1$ first trimester, $T 2$ second trimester

${ }^{a}$ Only mean follow up is reported

"Excludes those who had completion hysterectomy, or received fertility-compromising adjuvant treatment

\& Does not include ongoing pregnancies 
Table 8 Oncologic outcomes of fertility-sparing surgery after NACT (where $N>5$ reported)

\begin{tabular}{|c|c|c|c|c|c|c|c|}
\hline Study & $\begin{array}{l}\mathrm{N} \text { who } \\
\text { received NACT }\end{array}$ & $\begin{array}{l}\text { Timing of } L N \\
\text { assessment } \\
\text { (N positive } L N)\end{array}$ & $\begin{array}{l}\mathrm{NACT} \\
\text { regimen }\end{array}$ & $\begin{array}{l}\text { Surgical } \\
\text { procedure }\end{array}$ & $\begin{array}{l}N \text { recurrence } \\
\text { (mos) }\end{array}$ & $\begin{array}{l}\mathrm{N} \text { dead of } \\
\text { disease (mos) }\end{array}$ & $\begin{array}{l}\text { Median follow up } \\
\text { months for entire } \\
\text { series (range) }\end{array}$ \\
\hline Maneo 2008 [65] & 21 & After NACT (2) & TIP/TEP $\times 3$ & Cone & $0^{a}$ & 0 & $69(10-124)$ \\
\hline Robova 2010 [132] & 15 & After NACT (1) & TI/TAX3 & ST & $3(-)$ & $1(-)$ & $76.5(17-142)$ \\
\hline Marchiole 2011 [64] & 7 & After NACT (0) & TIP/TEP $\times 2-3$ & VRT & 0 & 0 & $22(5-49)$ \\
\hline $\begin{array}{l}\text { Vercecllino } 2012 \\
\text { [133] }\end{array}$ & 6 & Before NACT (0) & $\begin{array}{l}1-\mathrm{TP} \\
5-\mathrm{TIP}\end{array}$ & VRT & 0 & 0 & $30.6(8-70)$ \\
\hline $\begin{array}{l}\text { Lanowska } 2014 \\
\text { [134] }\end{array}$ & 20 & Before NACT (0) & $\mathrm{TIP} / \mathrm{TP} \times 2-3$ & VRT & $1(20)$ & 0 & $23(1-88)$ \\
\hline Salihi 2015 [131] & 11 & Before NACT (1) & $\begin{array}{l}2 \mathrm{TIP} \times 3 \\
4 \mathrm{ddCP} \times 3 \\
5 \mathrm{wCP} \times 3\end{array}$ & Cone & $1(40)$ & 0 & $58(13-122)$ \\
\hline Total & $N=80$ & & & & $N=5$ & $N=1$ & \\
\hline Crude rates (\%) & & & & & Recurrence rate $=6.3 \%^{\#}$ & Mortality rate $=1.3 \%{ }^{\#}$ & \\
\hline
\end{tabular}

Abbreviations: NACT neoadjuvant chemotherapy, LN lymph node, ST simple trachelectomy, VRT vaginal radical trachelectomy, TP cisplatin + paclitaxel, TI cisplatin + ifosfamide, TA cisplatin + doxorubicin (for adenocarcinoma), TIP cisplatin + paclitaxel + ifosfamide, TEP cisplatin + paclitaxel + epirubicin (for adenocarcinoma), ddCP dose dense carboplatin + paclitaxel, $w C P$ weekly carboplatin + paclitaxel

${ }^{\#}$ Crude recurrence and mortality rates among those who successfully underwent fertility sparing surgery, notwithstanding adjuvant treatment received

${ }^{a} N=3$ patients developed $\mathrm{CIN}$ in the residual cervix

showed higher rates of recurrence among a subset of women with positive nodes, in whom fertility-sparing surgery was aborted, compared to women with negative nodes that went on to have NACT and VRT. They suggested that nodal assessment prior to NACT identifies a high-risk group in whom fertility preservation should be avoided [43]. Conversely, some contend that the use of NACT prior to lymph node assessment in these patients may result in fewer nodal metastases, and thus a higher number of patients eligible for fertility-sparing surgery [43]. Our review of the literature identified only 3 series where nodal assessment was carried out prior to NACT (Table 6). While nodal involvement is one of the most significant negative prognostic factors in early-stage cervical cancer, up-front lymph node assessment could theoretically be used to tailor NACT regimen, rather than to exclude potential candidates for fertility-sparing surgery [43].

\section{Advanced-stage disease}

For patients who require hysterectomy and/or pelvic radiotherapy, fertility preservation depends on assisted reproductive technologies. Recognized options include oocyte or embryo cryopreservation prior to cancer therapy and ovarian transposition [5, 67]. The American Society for Reproductive Medicine in 2013 argues that given similar fertilization and pregnancy rates to IVF/ ICSI with fresh oocytes, oocyte cryopreservation should no longer be considered experimental [68]. Fertility preservation options that are still considered investigations include ovarian tissue cryopreservation/transplantation and uterine transplantation $[5,69,70]$.

The estimated lethal radiation dose to destroy $50 \%$ of oocytes is $\leq 2$ Gy [71] and dependent on age, doses as low as 6 Gy can render a woman menopausal, [72, 73]. The uterus also undergoes irreversible damage after

Table 9 Obstetrical outcomes of fertility-sparing surgery after NACT (where $N>5$ reported)

\begin{tabular}{llllll}
\hline Study & $\begin{array}{l}\text { Successful fertility } \\
\text { sparing management }\end{array}$ & Conceptions & T1/T2 losses & $\begin{array}{l}\text { Live births } \\
\text { (ongoing) }\end{array}$ & $\begin{array}{l}\text { Median follow up } \\
\text { months for entire } \\
\text { series (range) }\end{array}$ \\
\hline Maneo 2008 [65] & 16 & 10 & 1 & 9 & $69(10-124)$ \\
Robova 2010 [132] & 12 & 7 & 0 & $6(1)$ & $76.5(17-142)$ \\
Marchiole 2011 [64] & 7 & 1 & 0 & $0(1)$ & $22(5-49)$ \\
Lanowska 2014 [134] & 18 & 7 & 2 & $4(1)$ & 7 \\
Salihi 2015 [131] & 9 & 11 & 4 & $7-88)$ \\
Total & 62 & 36 & 7 & $26(3)$ & $58(13-122)$ \\
Crude rates (\%) & & & $19.4 \%$ T1/T2 loss rate & $72.2 \%$ live birth rate \\
\hline
\end{tabular}

Abbreviations: $T 1$ first trimester, $T 2$ second trimester

\#Excludes those who had completion hysterectomy, or received fertility-compromising adjuvant treatment

\& Does not include ongoing pregnancies 
doses from 14 to 30 Gy via reduced uterine volume, reduced elasticity of the uterine musculature and uterine vascular damage [71, 74].

Ovarian transposition was developed as a method to protect the ovaries from the effects of radiation. Due to the theoretical risk of remigration of the ovaries, the ASCO recommendations on fertility preservation suggest performing the transposition as close to the radiation treatment date as possible [5]. Even after the ovaries are transposed, short-term hormonal function is preserved in only approximately $50-93 \%$, with failure likely related to radiation scatter, remigration, and compromised ovarian blood supply [5, 75-80]. For all of the above reasons, we feel it is important that Gynecologic Oncologists familiar with the radiation borders perform such surgeries. Hwang et. al demonstrated that transposing the ovaries more than $1.5 \mathrm{~cm}$ above the iliac crest was significantly associated with successful preservation of ovarian function after treatment [81]. Despite increased success with higher fixation, the Royal College of Obstetricians and Gynecologists recommends that oocyte retrieval be considered for cervical cancer patients prior to the administration of radiation therapy due to the significant risk of ovarian failure after transposition [82]. New random-start ovarian hyperstimulation protocols decrease total time for the IVF cycle without compromising oocyte yield and maturity [83]. Apart from the risk of failure of ovarian transposition, there is also a concern about the risk of metastases in transposed ovaries [84-86]. Given that oophorectomy is not part of the standard treatment of cervical cancer, this risk is not considered prohibitive. Regardless, it seems that ovarian transposition is provided to only a small fraction of eligible patients [87]. A study Salih et. al. in 2015 also suggested that few cervical cancer patients who undergo ovarian transposition end up pursuing in vitro fertilization [88]. The reality is that patients who undergo ovarian transposition require a gestational carrier, which is fraught with ethical, financial and legal issues [89]. Although successful pregnancies have been reported for cervical cancer patients after oocyte retrieval from transposed ovaries and transfer to a gestational carrier, these reports are rare [90-92]. It seems that for most women, the main benefit of ovarian transposition is maintenance of hormonal function rather than preservation of fertility.

\section{Experimental technique for fertility preservation}

Ovarian tissue cryopreservation is an experimental technique for fertility preservation. It is sometimes offered to patients who require immediate gonadotoxic treatment of malignancies where there is insufficient time to offer ovulation induction and cryopreservation of oocytes or embryos. Ovarian tissue can be cryopreserved as cortical biopsies, cortical strips or as whole ovaries, but only cortical biopsies and strips have been successfully transplanted after cryopreservation [69]. The ovarian tissue can be transplanted into a pelvic (orthotopic) or extrapelvic (heterotopic) site. When cortical strips are transplanted orthotopically, they are transplanted either into the medulary portion of a remaining ovary or onto the peritoneum of the ovarian fossa [69]. Studies have reported normal menstrual cycles within 4-9 months after transplantation and graft survival from several months up to 7 years $[69,93-96]$. There have been at least 24 births reported after orthotopic transplantation of cortical ovarian tissue, but many of these reports are confounded by the presence of native ovarian tissue [69, 95, 97-99]. Locations of heterotopic transplantation of ovarian tissue resulting in restoration of ovarian function include the forearm, abdominal wall and chest wall [69, 100-102]. Although oocyte retrieval and fertilization with IVF have been reported, there have been no reported live births with this technique. The risk of malignancy from autotransplanted ovarian tissue in cancer patients is not clear, but in a systematic review of 289 patients, metastases were common in patients with leukemia, but less common in most other cancers including cervical cancer [103].

Another experimental procedure that has had recent media attention is uterine transplantation. A 1-year follow-up report of the first uterine transplant trial was published in 2015 [104]. The trial involved uterine transplantation in 9 patients with normal ovarian function who had previously undergone IVF and had cryopreserved embryos. One patient had a history of hysterectomy for cervical cancer. Of the 9 transplanted uteri, 2 were removed within the first 6 months, one due to chronic infection and the other due to bilateral uterine vessel thrombosis. In addition to these grade III surgical complications in the recipients, one of the donors developed a ureterovaginal fistula. Participants were placed on an immunosuppressive protocol. After 1 year of follow-up, 5 of the 7 recipients who kept their transplanted uterus experienced rejection episodes which were all asymptomatic and managed with an intensification of the immunosuppressive regimen. Spontaneous menses occurred in all 7 women within 2 months of transplantation. The plan was to transfer embryos after 12-18 months post-transplant, and a 4-6 month rejection-free period, and to remove the uterus after 1-2 successful pregnancies. As of December, 2015 there were apparently 4 healthy babies born to this cohort [70].

\section{Patient reported outcomes after fertility-sparing management}

While we have made impressive technological advances in early cervical cancer management, the long-term effects of cancer survivorship on quality of life are still 
not fully understood [105]. Several prospective observational studies have assessed patient reported outcomes after fertility-sparing surgery in early cervical cancer. In 2010, Carter et al. conducted a 2-year prospective study assessing the emotional, sexual, and quality of life concerns of women undergoing radical trachelectomy versus radical hysterectomy for treatment of early-stage cervical cancer [105]. Pre-operatively, both groups reported increased depression, distress, and sexual dysfunction. Although these measurements improved over time, they did not differ significantly by surgery type. This highlights the challenges faced by young cancer survivors in general. After a few years of procedural experience with non-radical fertility-sparing surgery, Song et al., in 2013, examined the effects of surgical radicality on sexual functioning [106]. Women who had undergone non-radical surgery experienced less sexual dysfunction than those who had undergone radical surgery. There was no difference between those who had undergone radical trachelectomy versus radical hysterectomy [106]. While these non-randomized studies generate important hypotheses, they are limited by small sample sizes.

In addition to GOG 278, a prospective questionnairebased study is currently assessing quality of life and sexual function in women who have undergone radical abdominal trachelectomy [107]. It is expected that these studies will shed light on the patient experience, both in terms of fertility-sparing and non-radical management of early-stage cervical cancer.

\section{Conclusions}

Today, many cervical cancer survivors have the option of becoming a parent. For those with early-stage disease, minimally invasive and fertility sparing techniques have resulted in improved obstetrical outcomes without compromising oncologic safety. For others, natural fertility and childbearing may be unachievable. However, advances in assisted reproductive technologies continue to make pregnancy and/or parenthood a possibility for those who desire it.

Several questions still remain. The safety of non-radical, fertility-sparing surgery has mainly been demonstrated in the context of small, non-randomized comparisons that are fraught with selection bias. The appropriate timing of NACT with respect to nodal assessment, in this context, has yet to be elucidated. The significance of nodal micrometastases remains unclear. For those fortunate enough to undergo fertility preservation and achieve pregnancy, management should be standardized. Centres of excellence should be established, involving gynecologic oncologists, reproductive endocrinologists, maternal fetal medicine specialists, and psychologists specializing in sexual and reproductive health. Ultimately, as we continue to seek answers to our objective questions, the patientcentered purpose of this quest should not be forgotten.

\section{Abbreviations}

AC: Adenocarcinoma; ART: Abdominal radical trachelectomy; AS: Adenosquamous carcinoma; ASCO: American Society of Clinical Oncology; CT: Computed tomography; Cx bx: Cervical biopsy; FIGO: International Federation of Gynecology and Obstetrics; GOG: Gynecologic Oncology Group; Gy: Gray; ICSI: Intracytoplasmic sperm injection; IVF: In vitro fertilization; LARVT: Laparoscopic-assisted vaginal radical trachelectomy; LEEP: Loop electrical excision procedure; LN: Lymph node; LRT: Laparoscopic radical trachelectomy; LVSI: Lymphovascular space invasion; MRI: Magnetic resonance imaging; NACT: Neoadjuvant chemotherapy; NCCN: National Comprehensive Cancer Network; PET$C T$ : Positron emission tomography-computed tomography; RRT: Robotic radical trachelectoomy; RT: Radical trachelectomy; SCC: Squamous cell carcinoma; SEER: Surveillance, Epidemiology, and End Results database; SLNP: Sentinel lymph node procedure; ST: Simple trachelectomy; T1: First trimester; T2: Second trimester; VRT: Vaginal radical trachelectomy

\section{Acknowledgments \\ None.}

Funding

No funding was specifically obtained for this review.

Availability of data and materials

Not applicable (all data from previously published sources, see references).

Authors' contributions

KW was involved in data acquisition, analysis, and interpretation, and was responsible for drafting $>80 \%$ of the manuscript. GL was also involved in data acquisition, and interpretation, drafting parts of the review, and revisions of the final manuscript. AC was involved in conception and design of the review, revisions of the manuscript and provided content expertise throughout. All authors gave final approval for publication.

\section{Competing interests}

The authors declare that they have no competing interests.

Consent for publication

Not applicable.

Ethics approval and consent to participate

Not applicable.

Received: 15 June 2016 Accepted: 27 September 2016

Published online: 21 October 2016

\section{References}

1. Ferlay J, Soerjomataram I, Dikshit R, Eser S, Mathers C, Rebelo M, et al. Cancer incidence and mortality worldwide: sources, methods and major patterns in GLOBOCAN 2012. Int J Cancer. 2015;136(5):E359-86.

2. Howlader N NA, Krapcho M, Garshell J, Miller D, Altekruse SF, Kosary CL, Yu M, Ruhl J, Tatalovich Z,Mariotto A, Lewis DR, Chen HS, Feuer EJ, Cronin KA (eds). . SEER Cancer Statistics Review, 1975-2012. Bethesda, MD: National Cancer Institute., 2015.

3. Carter J, Rowland K, Chi D, Brown C, Abu-Rustum N, Castiel M, et al. Gynecologic cancer treatment and the impact of cancer-related infertility. Gynecol Oncol. 2005;97(1):90-5.

4. Wenzel L, Dealba I, Habbal R, Kluhsman BC, Fairclough D, Krebs LU, et al. Quality of life in long-term cervical cancer survivors. Gynecol Oncol. 2005;97(2):310-7.

5. Lee SJ, Schover LR, Partridge AH, Patrizio P, Wallace WH, Hagerty K, et al. American Society of Clinical Oncology recommendations on fertility preservation in cancer patients. J Clin Oncol Off J Am Soc Clin Oncol. 2006;24(18):2917-31.

6. Capilna ME, loanid N, Scripcariu V, Gavrilescu MM, Szabo B. Abdominal radical trachelectomy: a Romanian series. Int J Gynecol Cancer Off J Int Gynecol Cancer Soc. 2014;24(3):615-9. 
7. Dargent DBJ, Roy M, et al. La trachelectomie elargie (TE) une alternative a I'hysterectomie radicale dans le traitement des cancers infiltrants developes sur la face externe du col uterin. J Obstet Gynaecol. 1994;2:285-92.

8. Sagae S, Monk BJ, Pujade-Lauraine E, Gaffney DK, Narayan K, Ryu SY, et al. Advances and Concepts in Cervical Cancer Trials: A Road Map for the Future. Int J Gynecol Cancer Off J Int Gynecol Cancer Soc. 2015;26:199-207.

9. Koh WJ, Greer BE, Abu-Rustum NR, Apte SM, Campos SM, Cho KR, et al. Cervical Cancer, Version 2.2015. Journal of the National Comprehensive Cancer Network : JNCCN. 2015;13:395-404; quiz

10. Salicru SR, de la Torre JF, Gil-Moreno A. The surgical management of earlystage cervical cancer. Curr Opin Obstet Gynecol. 2013;25(4):312-9.

11. Reade CJ, Eiriksson LR, Covens A. Surgery for early stage cervical cancer: how radical should it be? Gynecol Oncol. 2013;131(1):222-30.

12. Covens A, Rosen B, Murphy J, Laframboise S, Depetrillo AD, Lickrish G, et al. Changes in the demographics and perioperative care of stage IA (2)/IB (1) cervical cancer over the past 16 years. Gynecol Oncol. 2001;81(2):133-7.

13. Comerci G, Bolger BS, Flannelly G, Maini M, de Barros LA, Monaghan JM. Prognostic factors in surgically treated stage IB-IIB carcinoma of the cervix with negative lymph nodes. Int J Gynecol Cancer Off J Int Gynecol Cancer Soc. 1998;8(1):23-6.

14. Quinn MA, Benedet JL, Odicino F, Maisonneuve P, Beller U, Creasman WT, et al. Carcinoma of the cervix uteri. FIGO 26th Annual Report on the Results of Treatment in Gynecological Cancer. International journal of gynaecology and obstetrics: the official organ of the International Federation of Gynaecology and Obstetrics. 2006;95 Suppl 1:S43-103.

15. Pikaart DP, Holloway RW, Ahmad S, Finkler NJ, Bigsby GE, Ortiz BH, et al. Clinical-pathologic and morbidity analyses of Types 2 and 3 abdominal radical hysterectomy for cervical cancer. Gynecol Oncol. 2007;107(2):205-10.

16. Alexander-Sefre F, Chee N, Spencer C, Menon U, Shepherd JH. Surgical morbidity associated with radical trachelectomy and radical hysterectomy. Gynecol Oncol. 2006;101(3):450-4.

17. Covens A, Rosen B, Gibbons A, Osborne R, Murphy J, Depetrillo A, et al, Differences in the morbidity of radical hysterectomy between gynecological oncologists. Gynecol Oncol. 1993;51(1):39-45.

18. Pieterse QD, Maas CP, ter Kuile MM, Lowik M, van Eijkeren MA, Trimbos JB, et al. An observational longitudinal study to evaluate miction, defecation, and sexual function after radical hysterectomy with pelvic lymphadenectomy for early-stage cervical cancer. Int J Gynecol Cancer Off J Int Gynecol Cancer Soc. 2006;16(3):1119-29.

19. Bergmark K, Avall-Lundqvist E, Dickman PW, Henningsohn L, Steineck G. Vaginal changes and sexuality in women with a history of cervical cancer. $N$ Engl J Med. 1999;340(18):1383-9.

20. Sood AK, Nygaard I, Shahin MS, Sorosky Jl, Lutgendorf SK, Rao SS. Anorectal dysfunction after surgical treatment for cervical cancer. J Am Coll Surg. 2002;195(4):513-9.

21. Landoni F, Maneo A, Zapardiel I, Zanagnolo V, Mangioni C. Class I versus class III radical hysterectomy in stage IB1-IIA cervical cancer. A prospective randomized study. Eur J Surg Oncol J Eur Soc Surg Oncol Br Assoc Surg Oncol. 2012:38(3):203-9.

22. Landoni F, Maneo A, Cormio G, Perego P, Milani R, Caruso O, et al. Class II versus class III radical hysterectomy in stage IB-IIA cervical cancer: a prospective randomized study. Gynecol Oncol. 2001;80(1):3-12.

23. Ribeiro Cubal AF, Ferreira Carvalho Jl, Costa MF, Branco AP. Fertility-sparing surgery for early-stage cervical cancer. International journal of surgical oncology. 2012;2012:936534.

24. Jacobsson B, Ladfors L, Milsom I. Advanced maternal age and adverse perinatal outcome. Obstet Gynecol. 2004;104(4):727-33.

25. Delgado G, Bundy BN, Fowler Jr WC, Stehman FB, Sevin B, Creasman WT, et al. A prospective surgical pathological study of stage I squamous carcinoma of the cervix: a Gynecologic Oncology Group Study. Gynecol Oncol. 1989;35(3):314-20.

26. Peters 3rd WA, Liu PY, Barrett 2nd RJ, Stock RJ, Monk BJ, Berek JS, et al. Concurrent chemotherapy and pelvic radiation therapy compared with pelvic radiation therapy alone as adjuvant therapy after radical surgery in high-risk early-stage cancer of the cervix. J Clin Oncol Off J Am Soc Clin Oncol. 2000;18(8):1606-13.

27. Sedlis A, Bundy BN, Rotman MZ, Lentz SS, Muderspach LI, Zaino RJ. A randomized trial of pelvic radiation therapy versus no further therapy in selected patients with stage IB carcinoma of the cervix after radical hysterectomy and pelvic lymphadenectomy: A Gynecologic Oncology Group Study. Gynecol Oncol. 1999;73(2):177-83.
28. Mathevet $P$, Laszlo de Kaszon E, Dargent D. Fertility preservation in early cervical cancer]. Gynecol Obstet Fertil. 2003;31(9):706-12.

29. Hertel H, Kohler C, Grund D, Hillemanns P, Possover M, Michels W, et al. Radical vaginal trachelectomy (RVT) combined with laparoscopic pelvic lymphadenectomy: prospective multicenter study of 100 patients with early cervical cancer. Gynecol Oncol. 2006;103(2):506-11.

30. Plante M, Renaud MC, Francois H, Roy M. Vaginal radical trachelectomy: an oncologically safe fertility-preserving surgery. An updated series of 72 cases and review of the literature. Gynecol Oncol. 2004;94(3):614-23.

31. Schlaerth JB, Spirtos NM, Schlaerth AC. Radical trachelectomy and pelvic lymphadenectomy with uterine preservation in the treatment of cervical cancer. Am J Obstet Gynecol. 2003;188(1):29-34.

32. Peppercorn PD, Jeyarajah AR, Woolas R, Shepherd JH, Oram DH, Jacobs IJ, et al. Role of MR imaging in the selection of patients with early cervical carcinoma for fertility-preserving surgery: initial experience. Radiology. 1999;212(2):395-9.

33. Gien LT, Beauchemin MC, Thomas G. Adenocarcinoma: a unique cervical cancer. Gynecol Oncol. 2010;116(1):140-6.

34. Gien LT, Covens A. Fertility-sparing options for early stage cervical cancer. Gynecol Oncol. 2010;117(2):350-7.

35. Helpman L, Grisaru D, Covens A. Early adenocarcinoma of the cervix: is radical vaginal trachelectomy safe? Gynecol Oncol. 2011;123(1):95-8.

36. Touhami $\mathrm{O}$, Plante M. Should ovaries be removed or not in (early-stage) adenocarcinoma of the uterine cervix: a review. Gynecol Oncol. 2015;136(2):384-8.

37. Agarwal S, Schmeler KM, Ramirez PT, Sun CC, Nick A, Dos Reis R, et al. Outcomes of patients undergoing radical hysterectomy for cervical cancer of high-risk histological subtypes. Int J Gynecol Cancer Off J Int Gynecol Cancer Soc. 2011;21(1):123-7.

38. Beiner ME, Covens A. Surgery insight: radical vaginal trachelectomy as a method of fertility preservation for cervical cancer. Nat Clin Pract Oncol. 2007:4(6):353-61.

39. Marchiole P, Buenerd A, Benchaib M, Nezhat K, Dargent D, Mathevet P. Clinical significance of lympho vascular space involvement and lymph node micrometastases in early-stage cervical cancer: a retrospective case-control surgico-pathological study. Gynecol Oncol. 2005;97(3):727-32.

40. McCann GA, Taege SK, Boutsicaris CE, Phillips GS, Eisenhauer EL, Fowler JM, et al. The impact of close surgical margins after radical hysterectomy for early-stage cervical cancer. Gynecol Oncol. 2013;128(1):44-8.

41. Plante M, Roy M. New approaches in the surgical management of early stage cervical cancer. Curr Opin Obstet Gynecol. 2001;13(1):41-6.

42. Ismiil N, Ghorab Z, Covens A, Nofech-Mozes S, Saad R, Dube V, et al. Intraoperative margin assessment of the radical trachelectomy specimen. Gynecol Oncol. 2009;113(1):42-6.

43. Eiriksson L, Covens A. Advancing fertility-sparing treatments in cervical cancer: where is the limit? Gynecol Oncol. 2012;126(3):317-8.

44. Panici PB, Angioli R, Palaia I, Muzii L, Zullo MA, Manci N, et al. Tailoring the parametrectomy in stages IA2-IB1 cervical carcinoma: is it feasible and safe? Gynecol Oncol. 2005;96(3):792-8.

45. Scholz HS, Lax SF, Benedicic C, Tamussino K, Winter R. Accuracy of frozen section examination of pelvic lymph nodes in patients with FIGO stage IB1 to IIB cervical cancer. Gynecol Oncol. 2003;90(3):605-9.

46. Gortzak-Uzan L, Jimenez W, Nofech-Mozes S, Ismiil N, Khalifa MA, Dube V, et al. Sentinel lymph node biopsy vs. pelvic lymphadenectomy in early stage cervical cancer: is it time to change the gold standard? Gynecol Oncol. 2010;116(1):28-32

47. Gubbala PK, Laios A, Wang Z, Dhar S, Pathiraja PJ, Haldar K, et al. Routine Intraoperative Frozen Section Examination to Minimize Bimodal Treatment in Early-Stage Cervical Cancer. Int J Gynecol Cancer Off J Int Gynecol Cancer Soc. 2016;26(6):1148-53.

48. Lv X, Chen L, Yu H, Zhang X, Yan D. Intra-operative frozen section analysis of common iliac lymph nodes in patients with stage IB1 and IIA1 cervical cancer. Arch Gynecol Obstet. 2012;285(3):811-6.

49. Dursun $\mathrm{P}$, Leblanc $\mathrm{E}$, Nogueira MC. Radical vaginal trachelectomy (Dargent's operation): a critical review of the literature. Eur J Surg Oncol J Eur Soc Surg Oncol Br Assoc Surg Oncol. 2007;33(8):933-41.

50. Einstein MH, Park KJ, Sonoda Y, Carter J, Chi DS, Barakat RR, et al. Radical vaginal versus abdominal trachelectomy for stage IB1 cervical cancer: a comparison of surgical and pathologic outcomes. Gynecol Oncol. 2009;112(1):73-7.

51. Cao DY, Yang JX, Wu XH, Chen YL, Li L, Liu KJ, et al. Comparisons of vaginal and abdominal radical trachelectomy for early-stage cervical cancer: 
preliminary results of a multi-center research in China. $\mathrm{Br} J$ Cancer. 2013;109(11):2778-82.

52. Boss EA, van Golde RJ, Beerendonk CC, Massuger LF. Pregnancy after radical trachelectomy: a real option? Gynecol Oncol. 2005;99(3 Suppl 1):S152-6.

53. Li J, Li Z, Wang H, Zang R, Zhou Y, Ju X, et al. Radical abdominal trachelectomy for cervical malignancies: surgical, oncological and fertility outcomes in 62 patients. Gynecol Oncol. 2011;121(3):565-70.

54. Abu-Rustum NR, Neubauer N, Sonoda Y, Park KJ, Gemignani M, Alektiar KM, et al. Surgical and pathologic outcomes of fertility-sparing radical abdominal trachelectomy for FIGO stage IB1 cervical cancer. Gynecol Oncol. 2008;111(2):261-4

55. Bouchard-Fortier G, Reade CJ, Covens A. Non-radical surgery for small earlystage cervical cancer. Is it time? Gynecol Oncol. 2014;132(3):624-7.

56. Covens A, Rosen B, Murphy J, Laframboise S, Depetrillo AD, Lickrish G, et al. How important is removal of the parametrium at surgery for carcinoma of the cervix? Gynecol Oncol. 2002;84(1):145-9.

57. Plante M, Gregoire J, Renaud MC, Roy M. The vaginal radical trachelectomy: an update of a series of 125 cases and 106 pregnancies. Gynecol Oncol. 2011:121(2):290-7.

58. Lanowska M, Mangler M, Spek A, Grittner U, Hasenbein K, Chiantera V, et al. Radical vaginal trachelectomy (RVT) combined with laparoscopic lymphadenectomy: prospective study of 225 patients with early-stage cervical cancer. Int J Gynecol Cancer Off J Int Gynecol Cancer Soc. 2011;21(8):1458-64.

59. Shepherd JH. Cervical cancer. Best practice \& research Clinical obstetrics \& gynaecology. 2012;26(3):293-309.

60. Ramirez PT, Pareja R, Rendon GJ, Millan C, Frumovitz M, Schmeler KM Management of low-risk early-stage cervical cancer: should conization simple trachelectomy, or simple hysterectomy replace radical surgery as the new standard of care? Gynecol Oncol. 2014;132(1):254-9.

61. Radical Versus Simple Hysterectomy and Pelvic Node Dissection in Patients With Low-risk Early Stage Cervical Cancer (SHAPE). ClinicalTrials.gov Identifier: NCT01658930. https://clinicaltrials.gov/ct2/show/NCT01658930 Accessed 26 May 2016

62. Conservative Surgery for Women with Cervical Cancer. ClinicalTrials.gov Identifier: NCT01048853. https://clinicaltrials.gov/ct2/show/NCT01048853. Accessed 26 May 2016.

63. Studying the Physical Function and Quality of Life Before and After Surgery in Patients With Stage 1 Cervical Cancer. ClinicalTrials.gov Identifier: NCT01649089. https://clinicaltrials.gov/ct2/show/NCT01649089. Accessed 26 May 2016.

64. Marchiole P, Tigaud JD, Costantini S, Mammoliti S, Buenerd A, Moran E, et al. Neoadjuvant chemotherapy and vaginal radical trachelectomy for fertility-sparing treatment in women affected by cervical cancer (FIGO stage IB-IIA1). Gynecol Oncol. 2011;122(3):484-90.

65. Maneo A, Chiari S, Bonazzi C, Mangioni C. Neoadjuvant chemotherapy and conservative surgery for stage IB1 cervical cancer. Gynecol Oncol. 2008;111(3):438-43.

66. Kim HS, Sardi JE, Katsumata N, Ryu HS, Nam JH, Chung HH, et al. Efficacy of neoadjuvant chemotherapy in patients with FIGO stage IB1 to IIA cervical cancer: an international collaborative meta-analysis. Eur J Surg Oncol J Eur Soc Surg Oncol Br Assoc Surg Oncol. 2013;39(2):115-24.

67. ACOG. Committee Opinion No. 584: oocyte cryopreservation. Obstet Gynecol. 2014;123(1):221-2.

68. Pfeifer S, Goldberg J, McClure R, Lobo R, Thomas M, Widra E, Licht M, Collins J, Cedars M, Racowsky C, Vernon M, Davis O, Gracia C, Catherino W, Thornton K, Rebar R, La Barbera A. Mature oocyte cryopreservation: a guideline. Fertility and sterility. 2013;99(1):37-43.

69. Pfeifer S, Goldberg J, Lobo R, Pisarska M, Thomas M, Widra E, Sandlow J, Licht M, Rosen M, Vernon M, Catherino W, Davis O, Dumesic D, Gracia C, Odem R, Thornton K, Reindollar R, Rebar R, La Barbera A. Ovarian tissue cryopreservation: a committee opinion. Fertility and sterility. 2014;101(5): $1237-43$.

70. Brannstrom M. Uterus transplantation. Current opinion in organ transplantation. 2015;20(6):621-8.

71. Wallace WH, Thomson AB, Kelsey TW. The radiosensitivity of the human oocyte. Human reproduction (Oxford, England). 2003;18 (1):117-21.

72. Lushbaugh CC, Casarett GW. The effects of gonadal irradiation in clinical radiation therapy: a review. Cancer. 1976;37(2 Suppl):1111-25.

73. Ghadjar P, Budach V, Kohler C, Jantke A, Marnitz S. Modern radiation therapy and potential fertility preservation strategies in patients with cervical cancer undergoing chemoradiation. Radiation oncology (London, England). 2015;10:50

74. Critchley HO, Wallace WH, Shalet SM, Mamtora H, Higginson J, Anderson DC. Abdominal irradiation in childhood; the potential for pregnancy. Br J Obstet Gynaecol. 1992;99(5):392-4.

75. Clough KB, Goffinet F, Labib A, Renolleau C, Campana F, de la Rochefordiere A, et al. Laparoscopic unilateral ovarian transposition prior to irradiation: prospective study of 20 cases. Cancer. 1996;77(12):2638-45.

76. Barahmeh S, Al Masri M, Badran O, Masarweh M, El-Ghanem M, Jaradat I, et al. Ovarian transposition before pelvic irradiation: indications and functional outcome. J Obstet Gynaecol Res. 2013;39(11):1533-7.

77. Olejek A, Wala D, Chimiczewski P, Rzempoluch J. Hormonal activity of transposed ovaries in young women treated for cervical cancer. Int J Gynecol Cancer Off J Int Gynecol Cancer Soc. 2001;15(1):5-13.

78. Huang KG, Lee CL, Tsai CS, Han CM, Hwang LL. A new approach for laparoscopic ovarian transposition before pelvic irradiation. Gynecol Oncol. 2007:105(1):234-7.

79. Ishii K, Aoki Y, Takakuwa K, Tanaka K. Ovarian function after radical hysterectomy with ovarian preservation for cervical cancer. J Reprod Med. 2001;46(4):347-52.

80. Pahisa J, Martinez-Roman S, Martinez-Zamora MA, Torne A, Caparros X, Sanjuan A, et al. Laparoscopic ovarian transposition in patients with early cervical cancer. Int J Gynecol Cancer Off J Int Gynecol Cancer Soc. 2008;18(3):584-9.

81. Hwang JH, Yoo HJ, Park SH, Lim MC, Seo SS, Kang S, et al. Association between the location of transposed ovary and ovarian function in patients with uterine cervical cancer treated with (postoperative or primary) pelvic radiotherapy. Fertility and sterility. 2012:97 (6):1387-93.e1-2.

82. Farthing AG-MS. Fertility Sparing Treatments in Gynaecological Cancers RCOG Guidelines: Royal College of Obstetricians and Gynaecologists, 2013 February, 2013. Report No.: 35 Contract No.: 35.

83. Cakmak H, Rosen MP. Random-start ovarian stimulation in patients with cancer. Curr Opin Obstet Gynecol. 2015;27(3):215-21.

84. Delotte J, Ferron G, Kuei TL, Mery E, Gladieff L, Querleu D. Laparoscopic management of an isolated ovarian metastasis on a transposed ovary in a patient treated for stage IB1 adenocarcinoma of the cervix. J Minim Invasive Gynecol. 2009:16(1):106-8.

85. Shigematsu T, Ohishi Y, Fujita T, Higashihara J, Irie T, Hayashi T. Metastatic carcinoma in a transposed ovary after radical hysterectomy for a stage 1B cervical adenosquamous cell carcinoma. Case report. Eur J Gynaecol Oncol. 2000;21(4):383-6.

86. Morice P, Haie-Meder C, Pautier P, Lhomme C, Castaigne D. Ovarian metastasis on transposed ovary in patients treated for squamous cell carcinoma of the uterine cervix: report of two cases and surgical implications. Gynecol Oncol. 2001;83(3):605-7.

87. Han SS, Kim YH, Lee SH, Kim GJ, Kim HJ, Kim JW, et al. Underuse of ovarian transposition in reproductive-aged cancer patients treated by primary or adjuvant pelvic irradiation. J Obstet Gynaecol Res. 2011;37(7):825-9.

88. Salih SM, Albayrak S, Seo S, Stewart SL, Bradley K, Kushner DM. Diminished Utilization of in Vitro Fertilization Following Ovarian Transposition in Cervical Cancer Patients. J Reprod Med. 2015:60:345-53.

89. Brezina PR, Zhao Y. The ethical, legal, and social issues impacted by modern assisted reproductive technologies. Obstetrics and gynecology international. 2012;2012:686253.

90. Giacalone PL, Laffargue F, Benos P, Dechaud H, Hedon B. Successful in vitro fertilization-surrogate pregnancy in a patient with ovarian transposition who had undergone chemotherapy and pelvic irradiation. Fertil Steril. 2001;76(2): 388-9.

91. Zinger M, Liu JH, Husseinzadeh N, Thomas MA. Successful surrogate pregnancy after ovarian transposition, pelvic irradiation and hysterectomy. J Reprod Med. 2004;49(7):573-4.

92. Agorastos T, Zafrakas M, Mastrominas M. Long-term follow-up after cervical cancer treatment and subsequent successful surrogate pregnancy. Reprod Biomed Online. 2009:19(2):250-1.

93. Donnez J, Dolmans MM, Demylle D, Jadoul P, Pirard C, Squifflet J, et al. Livebirth after orthotopic transplantation of cryopreserved ovarian tissue. Lancet (London, England). 2004;364 (9443):1405-10.

94. Radford JA, Lieberman BA, Brison DR, Smith AR, Critchlow JD, Russell SA, et al. Orthotopic reimplantation of cryopreserved ovarian cortical strips after 
high-dose chemotherapy for Hodgkin's lymphoma. Lancet (London, England). 2001;357 (9263):1172-5.

95. Schmidt KL, Andersen CY, Loft A, Byskov AG, Ernst E, Andersen AN. Followup of ovarian function post-chemotherapy following ovarian cryopreservation and transplantation. Human reproduction (Oxford, England). 2005;20 (12):3539-46.

96. Callejo J, Salvador C, Miralles A, Vilaseca S, Lailla JM, Balasch J. Long-term ovarian function evaluation after autografting by implantation with fresh and frozen-thawed human ovarian tissue. J Clin Endocrinol Metab. 2001;86(9):4489-94.

97. Grynberg M, Poulain M, Sebag-Peyrelevade S, le Parco S, Fanchin R, Frydman N. Ovarian tissue and follicle transplantation as an option for fertility preservation. Fertil Steril. 2012;97(6):1260-8.

98. Meirow D, Levron J, Eldar-Geva T, Hardan I, Fridman E, Zalel Y, et al. Pregnancy after transplantation of cryopreserved ovarian tissue in a patient with ovarian failure after chemotherapy. N Engl J Med. 2005;353(3):318-21.

99. Demeestere I, Simon P, Buxant F, Robin V, Fernandez SA, Centner J, et al. Ovarian function and spontaneous pregnancy after combined heterotopic and orthotopic cryopreserved ovarian tissue transplantation in a patient previously treated with bone marrow transplantation: case report. Human reproduction (Oxford, England). 2006;21 (8):2010-4.

100. Kim SS, Lee WS, Chung MK, Lee HC, Lee HH, Hill D. Long-term ovarian function and fertility after heterotopic autotransplantation of cryobanked human ovarian tissue: 8-year experience in cancer patients. Fertil Steril. 2009;91(6):2349-54.

101. Oktay K, Buyuk E, Veeck L, Zaninovic N, Xu K, Takeuchi T, et al. Embryo development after heterotopic transplantation of cryopreserved ovarian tissue. Lancet (London, England). 2004;363 (9412):837-40.

102. Rosendahl M, Loft A, Byskov AG, Ziebe S, Schmidt KT, Andersen AN, et al. Biochemical pregnancy after fertilization of an oocyte aspirated from a heterotopic autotransplant of cryopreserved ovarian tissue: case report. Human reproduction (Oxford, England). 2006;21 (8):2006-9.

103. Bastings $L$, Beerendonk CC, Westphal JR, Massuger LF, Kaal SE, van Leeuwen $\mathrm{FE}$, et al. Autotransplantation of cryopreserved ovarian tissue in cancer survivors and the risk of reintroducing malignancy: a systematic review. Hum Reprod Update. 2013;19(5):483-506.

104. Johannesson L, Kvarnstrom N, Molne J, Dahm-Kahler P, Enskog A, DiazGarcia C, et al. Uterus transplantation trial: 1-year outcome. Fertil Steril. 2015;103(1):199-204.

105. Carter J, Sonoda Y, Baser RE, Raviv L, Chi DS, Barakat RR, et al. A 2-year prospective study assessing the emotional, sexual, and quality of life concerns of women undergoing radical trachelectomy versus radical hysterectomy for treatment of early-stage cervical cancer. Gynecol Oncol. 2010;119(2):358-65.

106. Song T, Choi CH, Lee YY, Kim TJ, Lee JW, Kim BG, et al. Sexual function after surgery for early-stage cervical cancer: is there a difference in it according to the extent of surgical radicality? J Sex Med. 2012;9(6):1697-704.

107. Radical Trachelectomy for Cervical Cancer. ClinicalTrials.gov Identifier: NCT00813007. https://clinicaltrials.gov/ct2/show/NCT00813007. Accessed 26 May 2016

108. Shepherd JH, Spencer C, Herod J, Ind TE. Radical vaginal trachelectomy as a fertility-sparing procedure in women with early-stage cenvical cancer-cumulative pregnancy rate in a series of 123 women. BJOG. 2006;113(6):719-24.

109. Marchiole P, Benchaib M, Buenerd A, Lazlo E, Dargent D, Mathevet P. Oncological safety of laparoscopic-assisted vaginal radical trachelectomy (LARVT or Dargent's operation): a comparative study with laparoscopic-assisted vaginal radical hysterectomy (LARVH). Gynecol Oncol. 2007;106(1):132-41.

110. Wethington SL, Cibula D, Duska LR, Garrett L, Kim CH, Chi DS, et al. An international series on abdominal radical trachelectomy: 101 patients and 28 pregnancies. Int J Gynecol Cancer Off J Int Gynecol Cancer Soc. 2012;22(7):1251-7.

111. Mangler M, Lanowska M, Kohler C, Vercellino F, Schneider A, Speiser D. Pattern of cancer recurrence in 320 patients after radical vaginal trachelectomy. Int J Gynecol Cancer Off J Int Gynecol Cancer Soc. 2014;24(1):130-4.

112. Hauerberg L, Hogdall C, Loft A, Ottosen C, Bjoern SF, Mosgaard BJ, et al. Vaginal Radical Trachelectomy for early stage cervical cancer. Results of the Danish National Single Center Strategy. Gynecol Oncol. 2015;138:304-10.

113. Vieira MA, Rendon GJ, Munsell M, Echeverri L, Frumovitz M, Schmeler KM, et al. Radical trachelectomy in early-stage cervical cancer: A comparison of laparotomy and minimally invasive surgery. Gynecol Oncol. 2015;138:585-9.

114. Bernardini M, Barrett J, Seaward G, Covens A. Pregnancy outcomes in patients after radical trachelectomy. Am J Obstet Gynecol. 2003;189(5):1378-82.
115. Kim CH, Abu-Rustum NR, Chi DS, Gardner GJ, Leitao Jr MM, Carter J, et al. Reproductive outcomes of patients undergoing radical trachelectomy for early-stage cervical cancer. Gynecol Oncol. 2012;125(3):585-8.

116. Nishio H, Fujii T, Sugiyama J, Kuji N, Tanaka M, Hamatani T, et al. Reproductive and obstetric outcomes after radical abdominal trachelectomy for early-stage cervical cancer in a series of 31 pregnancies. Human reproduction (Oxford, England). 2013;28 (7):1793-8.

117. Kasuga $Y$, Nishio H, Miyakoshi K, Sato S, Sugiyama J, Matsumoto T, et al. Pregnancy Outcomes After Abdominal Radical Trachelectomy for EarlyStage Cervical Cancer: A 13-Year Experience in a Single Tertiary-Care Center. Int J Gynecol Cancer Off J Int Gynecol Cancer Soc. 2016;26(1):163-8.

118. Nishio H, Fujii T, Kameyama K, Susumu N, Nakamura M, Iwata T, et al. Abdominal radical trachelectomy as a fertility-sparing procedure in women with early-stage cervical cancer in a series of 61 women. Gynecol Oncol. 2009;115(1):51-5.

119. Li J, Wu X, Li X, Ju X. Abdominal radical trachelectomy: Is it safe for IB1 cervical cancer with tumors $>/=2 \mathrm{~cm}$ ? Gynecol Oncol. 2013;131(1):87-92

120. Lintner B, Saso S, Tarnai L, Novak Z, Palfalvi L, Del Priore G, et al. Use of abdominal radical trachelectomy to treat cervical cancer greater than $2 \mathrm{~cm}$ in diameter. Int J Gynecol Cancer Off J Int Gynecol Cancer Soc. 2013;23(6):1065-70.

121. Wethington SL, Sonoda Y, Park KJ, Alektiar KM, Tew WP, Chi DS, et al. Expanding the indications for radical trachelectomy: a report on 29 patients with stage IB1 tumors measuring 2 to $4 \mathrm{~cm}$. Int J Gynecol Cancer Off J Int Gynecol Cancer Soc. 2013;23(6):1092-8.

122. Bisseling KC, Bekkers RL, Rome RM, Quinn MA. Treatment of microinvasive adenocarcinoma of the uterine cervix: a retrospective study and review of the literature. Gynecol Oncol. 2007;107(3):424-30.

123. Rob L, Charvat M, Robova H, Pluta M, Strnad P, Hrehorcak M, et al. Less radical fertility-sparing surgery than radical trachelectomy in early cervical cancer. Int J Gynecol Cancer Off J Int Gynecol Cancer Soc. 2007;17(1):304-10.

124. Landoni F, Parma G, Peiretti M, Zanagnolo V, Sideri M, Colombo N, et al. Chemoconization in early cervical cancer. Gynecol Oncol. 2007;107(1 Suppl 1):S125-6.

125. Fagotti A, Gagliardi ML, Moruzzi C, Carone V, Scambia G, Fanfani F. Excisional cone as fertility-sparing treatment in early-stage cervical cancer. Fertil Steril. 2011;95(3):1109-12.

126. Maneo A, Sideri M, Scambia G, Boveri S, Dell'anna T, Villa M, et al. Simple conization and lymphadenectomy for the conservative treatment of stage IB1 cervical cancer. An Italian experience. Gynecol Oncol. 2011;123(3):557-60.

127. Raju SK, Papadopoulos AJ, Montalto SA, Coutts M, Culora G, Kodampur M, et al. Fertility-sparing surgery for early cervical cancer-approach to less radical surgery. Int J Gynecol Cancer Off J Int Gynecol Cancer Soc. 2012;22(2):311-7.

128. Palaia I, Musella A, Bellati F, Marchetti C, Di Donato V, Perniola G, et al. Simple extrafascial trachelectomy and pelvic bilateral lymphadenectomy in early stage cervical cancer. Gynecol Oncol. 2012;126(1):78-81.

129. Plante M, Gregoire J, Renaud MC, Sebastianelli A, Grondin K, Noel P, et al. Simple vaginal trachelectomy in early-stage low-risk cervical cancer: a pilot study of 16 cases and review of the literature. Int J Gynecol Cancer Off J Int Gynecol Cancer Soc. 2013;23(5):916-22.

130. Andikyan V, Khoury-Collado F, Denesopolis J, Park KJ, Hussein YR, Brown CL, et al. Cervical conization and sentinel lymph node mapping in the treatment of stage I cervical cancer: is less enough? Int I Gynecol Cancer Off J Int Gynecol Cancer Soc. 2014;24(1):113-7.

131. Salihi R, Leunen K, Van Limbergen E, Moerman P, Neven P, Vergote I. Neoadjuvant chemotherapy followed by large cone resection as fertilitysparing therapy in stage IB cervical cancer. Gynecol Oncol. 2015;139:447-51.

132. Robova H, Halaska M, Pluta M, Skapa P, Strnad P, Lisy J, et al. The role of neoadjuvant chemotherapy and surgery in cervical cancer. Int J Gynecol Cancer Off J Int Gynecol Cancer Soc. 2010;20(11 Suppl 2):S42-6.

133. Vercellino GF, Piek JM, Schneider A, Kohler C, Mangler M, Speiser D, et al. Laparoscopic lymph node dissection should be performed before fertility preserving treatment of patients with cervical cancer. Gynecol Oncol. 2012;126(3):325-9

134. Lanowska M, Mangler M, Speiser D, Bockholdt C, Schneider A, Kohler C, et al. Radical vaginal trachelectomy after laparoscopic staging and neoadjuvant chemotherapy in women with early-stage cervical cancer over $2 \mathrm{~cm}$ : oncologic, fertility, and neonatal outcome in a series of 20 patients. Int J Gynecol Cancer Off J Int Gynecol Cancer Soc. 2014;24(3):586-93. 